\title{
COMPLICATIONS OF MULTIPLE PREGNANCIES. OVERWIEW
}

\author{
S. Lazarov, L. Lazarov, N. Lazarov \\ Department of Obstetrics and Gynecology, Medical Faculty, Trakia University, Stara Zagora, Bulgaria
}

\begin{abstract}
Multiple gestations are high risk pregnancies which may be complicated by prematurity,low birthweight, preeclampsia, anaemia, postpartum haemorrhage, intrauterine growth restriction, neonatal morbidity and high neonatal and infant mortality. Preterm labor and birth represent the greatest risk to a multiple pregnancy. Sixty percent of multiples are born prematurely ( $<37$ weeks) compared to about $10 \%$ of singleton pregnancies. Placental function is more likely to be abnormal in a multiple pregnancy. Another placental problem is twintwin transfusion, a life-threatening condition in identical twins. Preeclampsia, also known as toxemia, occurs 2 to 5 times more often in multiple pregnancies. Fifteen percent to $20 \%$ of women with twin pregnancies will experience preeclampsia, and an even higher percentage is preeclamptic in triplet or high-order pregnancies. Preterm labor and birth pose the greatest risk to a multiple pregnancy. Sixty percent of multiples are born prematurely $(<37$ weeks) compared to about $10 \%$ of singleton pregnancies. Fetal and Newborn complications such as preterm delivery, respiratory distress syndrome, brain damage are responsible for almost $10 \%$ of premature newborn deaths. Birth defects and stillbirths account for about $30 \%$ of the deaths in twins and multiple pregnancies. Low birth weight of less than 5.5 pounds (lb.) [2,500 grams] occurs in over half of twins..
\end{abstract}

Key words: Preterm Birth, Placental Problems, Preeclampsia, Diabetes, TTTS (twin -twin transfusion syndrome).

Multiple gestations are high risk pregnancies which may be complicated by prematurity,low birthweight, pre-eclampsia, anaemia, postpartum haemorrhage, intrauterine growth restriction, neonatal morbidity and high neonatal and infant mortality.

Complications increase with each additional fetus in a multiple pregnancy and include many medical issues that will be discussed below. In addition to these, there is a higher incidence of severe nausea and vomiting, cesarean section, or forceps delivery. The pregnant women with twins or more should be aware of these and other potential problems. (1)

\section{Preterm Birth}

Preterm labor and birth pose the greatest risk to a multiple pregnancy. Sixty percent of multiples are born prematurely ( $<37$ weeks) compared to about $10 \%$ of singleton pregnancies. Feasibility of a vaginal delivery depends on the size, position, and health of the infants, as well as the size and shape of the mother's pelvic bones. Cesarean section is often needed for twin pregnancies and is expected for delivery of triplets.

The warning signs of early labor are: pelvic pressure, low back pain, increased vaginal discharge, or a change in the frequency of "false labor" pains should be reported to the physician. Sometimes, preterm delivery can be delayed by a few days or more if it is detected early. Each day gained provides valuable fetal growth and development. Once a woman is in advanced labor, delivery cannot be stopped. In rare instances, delivery of a second twin can be delayed. This delay, when possible, allows for continued growth in the protective environment of the uterus. Currently, there are no effective treatments to prevent preterm birth of multiples. (2).

\section{Placental Problems}

The placenta is attached to the wall of the uterus, and the fetus is attached to the placenta by the umbilical cord. The placenta provides blood, oxygen and nutrition to the fetus through the umbilical cord. 
Placental function is more likely to be abnormal in a multiple pregnancy. If the placenta is unable to provide adequate oxygen or nutrients to the fetus, the fetus cannot grow properly. Twins and high-order multiples that are more than $30 \%$ "underweight" by ultrasound measurements are at increased risk of complications and have death rates of nearly $25 \%$.

Another placental problem is twin-twin transfusion, a life-threatening condition in identical twins. This transfusion occurs when blood flows from one fetus to the other through a connection in a shared placenta. Poor growth occurs in the "donor" twin, and excessive fluid develops in the "recipient" twin. Therapeutic amniocentesis and laser coagulation of blood vessels that link circulation to the twins in the placenta(s) may reduce complications of twintwin transfusion. (3-9)

\section{Preeclampsia}

Preeclampsia, also known as toxemia, occurs 2 to 5 times more often in multiple pregnancies. Fifteen percent to $20 \%$ of women with twin pregnancies will experience preeclampsia, and an even higher percentage is preeclamptic in triplet or high-order pregnancies. Preeclampsia is diagnosed when the mother's blood pressure becomes elevated and protein is detected in the urine. The condition may progress and threaten the health of the mother and baby. When severe, the mother may have seizures, and stroke or other life-threatening complications are possible. (10)

\section{Diabetes}

Women with multiple pregnancies are more likely to develop gestational diabetes during pregnancy. Babies of diabetic mothers are more likely to experience respiratory distress and other newborn complications. However, gestational diabetes is common even in singleton pregnancies, and treatment is well established and effective.

\section{Fetal and Newborn Complications}

Preterm delivery place an infant at increased risk for severe complications or early death. A baby's lungs, brain, circulatory system, intestinal system, and eyes may be not fully developed.

Of the premature babies who die, $50 \%$ succumb to respiratory distress syndrome, caused by immature lungs. Brain damage is responsible for almost $10 \%$ of premature newborn deaths. (11)
LAZAROV S., et al.

Birth defects and stillbirths account for about $30 \%$ of the deaths in twins and multiple pregnancies (12). Neonatal intensive care unit admission is required for one-fourth of twin and three-fourths of triplet deliveries. (13)

Despite these numbers, it is important to note that the vast majority of multiple-birth infants do survive. Fetal death occurs in about $1.6 \%$ of twins and $2.7 \%$ of triplets. Furthermore, compared to singleton pregnancies of the same birth weight, there is no significant increase in the incidence of chronic lung disease or brain, eye, or gastrointestinal problems in multiplebirth infants. (14)

Low birth weight of less than 5.5 pounds (lb.) [2,500 grams] occurs in over half of twins. The average birth weight is approximately $4 \mathrm{lb}$. (1,660 grams) for triplets and $3 \mathrm{lb}$. (1,300 grams) for quadruplets. As a result of prematurity, the risk for cerebral palsy is 4 times more likely to occur in twins (15). The rates are even greater for triplets and highorder multiple births. The overall survival rate is $85 \%$ for newborns over $2 \mathrm{lb}, 3 \mathrm{oz}$. $(1,000$ grams) but less than $40 \%$ for those under $2 \mathrm{lb}$., $3 \mathrm{oz}$. Birth weight also corresponds closely to the severity of disability throughout the childhood years. Disability occurs in almost $25 \%$ of children with a birth weight less than 2 lb. $(16,17)$

\section{PREVENTION OF MULTIPLE PREGNANCY}

Prevention during infertility treatment is the best approach to avoiding a multiple pregnancy. In ART cycles, limiting the number of embryos transferred is an effective approach. In the United States, physicians and patients jointly decide how many embryos to transfer. However, in England, no more than two embryos may be transferred in most cases. In Canada, a maximum of three embryos are recommended for transfer. The ultimate goal of ART is to achieve a high pregnancy rate while transferring a single embryo. While physicians can transfer two embryos and still maintain acceptable pregnancy rates, the transfer of one embryo is associated with good pregnancy rates in certain patient groups, thereby resolving the problem of multiple pregnancies caused by multiple embryo transfer. Approximately $10 \%$ of embryo transfers in the United States are now performed using elective single embryo transfer. (18) 
Multiple pregnancies are a known complication of ovulation stimulation drugs. Most physicians monitor patients with ultrasound examinations and blood tests. A woman with a large number of ovarian follicles or high hormone levels has an increased risk of a multiple pregnancy, and the cycle may be canceled to avoid the risk.

\section{Multifetal Pregnancy Reduction}

When a triplet or high-order multiple pregnancy occurs, multifetal pregnancy reduction may be considered to improve the chance for survival of the fetuses. While multifetal pregnancy reduction carries some risk of a complete miscarriage, it also reduces the chances of extreme premature birth.

\section{Monitoring a Multiple Pregnancy}

Since preterm birth and growth disturbances are the major contributors to newborn death and disability in multiples, frequent obstetric visits and close monitoring of the pregnancy are needed.

Prenatal diagnosis using a variety of new techniques can be done near the end of the first trimester to screen for Down syndrome and other genetic abnormalities. Amniocentesis may be performed between 16 and 20 weeks. Amniocentesis may be complicated and difficult to perform in twins and triplets and may not be possible in high-order multiple pregnancies. However, reasonable data exist for the use of serum screening in the setting of multiple pregnancies and can be a helpful tool to assess risk of these and other conditions.

Many physicians perform cervical examinations every week or two beginning early in pregnancy to determine if the cervix is thinning or opening prematurely. If an exam or ultrasound shows that the cervix is thinning or beginning to dilate prematurely, a cerclage, or suture placed in the cervix, may prevent or delay premature dilatation. (19)

Tocolytic agents are medications that may slow or stop premature labor. It is important to attempt to delay delivery to minimize the risks of premature delivery. Ultrasound examinations in the second trimester can identify some birth defects. Assessment of fetal growth by ultrasound every 3 to 4 weeks during the second half of pregnancy is commonly performed.

Every multiple pregnancy should be considered at high risk.A neonatal intensive care unit nursery should be available to provide immediate and comprehensive support to premature newborns.

\section{Method of Delivery}

Vaginal delivery of twins may be safe in some circumstances. Many twins can be delivered vaginally if the lowest infant is in the head-first position. Most triplets will be delivered by cesarean section. Appropriate anesthesia and neonatal support are essential, whether delivery is performed vaginally or requires cesarean section. Delivery of multiples requires planning by the entire medical team and availability of full intensive-care support following birth. (20-24)

\section{CONCLUSION}

The objective of infertility treatment is the birth of a healthy child. In a small percentage of patients, treatment results in multiple pregnancy that may place the mother and the babies at increased risk for an unhealthy outcome. Since multiple pregnancies and their complications are an inevitable risk of fertility therapies, education about these risks is crucial prior to treatment. Ultimately, prevention is the key to reducing the risk of multiple pregnancy.

\section{REFERENCES}

1. N. Vassilev. Manuel of Obstetrics, Al. Hadjiev, N. Vassilev, 79-81. 1998.

2. R. Romero. Prediction of preterm delivery in twin gestations.

3. American society for reproductive medicine, Multiple Pregnancy and Birth: Twins, Triplets and High-order Multiples A Guide for Patients (Revised 2012).

4. R. Lenclen, G. Ciarlo, F. Proulx, Y. Ville. Echocardiographic assessment in preterm infants born from twin to twin transfusion syndrome treated by fetoscopic laser coagulation. 1-st World Congress - Twin pregnancy- a global perspective. 2009.

5. A. Fichera, M. Lanna, N. Fratelli, M. A. Rustico,T. Frusca, Feto-Maternal Unit, Spedali Civili, University of Brescia. Twin to twin transfusion syndrome presenting at early stages: is there still a possibile role for amnioreduction. 1-st World Congress Twin pregnancy- a global perspective. P 32. 2009.

6. J. Deprest, R. Devlieger, L. De Catte, L. Gucciardo, T. Van Mieghem, E. Done', L. Lewi. Losses following laser therapy for twin-to-twin transfusion syndrome. 1-st World Congress - Twin pregnancy- a global perspective. P 33. 2009. 
7. L. Gucciardo, P. Klaritsch, P. Lewi, et al. Middle cerebral artery peak systolic velocities in twin-to-twin transfusion syndrome fetuses around the time of fetoscopic laser coagulation. 1-st World Congress - Twin pregnancy- a global perspective. 2009.

8. Matias A. Is it feasible to screen for twintwin transfusion syndrome in the first trimester of pregnancy? what are the challenges and can they be met? 1-st World Congress - Twin pregnancy- a global perspective. 2009.

9. Y. Ville. Treatment of TTTS - 1-st World Congress - Twin pregnancy- a global perspective. 2009.

10.S. Stach, A.W. Liao, R. Assunc, a o, M. L. Brizot, M. Zugaib. Maternal morbidity in twin. pregnancies according to mode of delivery. 1-st World Congress - Twin pregnancy- a global perspective. P 103. 2009.

11.L. Sichinava, O. Panina, S. Kalashnikov, A. Povarova. Cervical length measurement in twin pregnancy: prediction of preterm delivery. 1-st World Congress - Twin pregnancy- a global perspective. P 97, 2009.

12.M. Vendemmia, S. Vendemmia1. Intrauterine death in multiple gestation: implications for surviving twin. 1-st World Congress - Twin pregnancy- a global perspective. P 115, 2009.

13.P. Vaas, K. Rull. Twin pregnancy: maternal risks, pregnancy complications, obstetric and perinatal outcome at tartu university hospitals women's clinic. (20032007). 1-st World Congress - Twin pregnancy- a global perspective. P 112. 2009.

14.A. Herbst, K. Ka“lle'n. Influence of deliverymodeonneonatal mortality for the second twin. 1-st World Congress - Twin pregnancy- a global perspective. P 48 . 2009.

15.V. Ciric ljubinkovic, J. Durutovic, N. Todorovic, O. Stanojlovic, M. Nikolic, J. Djorovic. Neonatal outcome of growth discordant twins. 1-st World Congress Twin pregnancy - a global perspective. 2009.
16.V. Sioulas, G. Salamalekis, S. Mourtzakis, T. Brozou et al. Growth discordance in twins: perinatal outcomes after elective cesarean section. 1-st World Congress Twin pregnancy - a global perspective. $\mathrm{P}$ 100. 2009.

17.M. D. Kilby. Mark D. Kilby. Professor of Fetal Medicine, Birmingham Women's Hospital, School of Clinical \& Experimental Medicine, College of Medicine, University of Birmingham, Birmingham, B15 2TG, United Kingdom. Fetal growth disorders in twins. 1-st World Congress - Twin pregnancy- a global perspective. 2009.

18.G. Monni, M.A. Zoppi. Phenotypic and genotypic discordance in monozygotic twins. 1-st World Congress - Twin pregnancy- a global perspective. 2009.

19. Cao A, Monni G. Phenotypic and genotypic discordance in monozygotic twins. In: Blickstein I, KeithLG . Multiple pregnancy. Epidemiology, gestation \&perinatal Outcome. 2nd ed. Taylor and Francis Group, New York; 2005. pp 226-32. 2009.

20.Franceschini P, Guala A, Vardeu MP, et al. Monozygotic twins and WiedemannBeckwith syndrome. Am J Med.Genet 1993;41:353-354. 2009.

21.B. Blondel, A. Macfarlane, M. Gissler, et al. and the PERISTAT Study Group. General Obstetrics BJOG 006;113:528-535. An international journal of Obstetrics and gynecology. Preterm birth and multiple pregnancy in European countries participating in the PERISTAT project.

22.Multiple Pregnancy and Birth: Twins, Triplets, and High-order Multiples. A Guide for Patients Revised 2012

23. Arihiro Shiozaki1, Satoshi Yoneda1, Masao Nakabayashi2, et al. Multiple pregnancy, short cervix, part-time worker, steroid use, low educational level and male fetus are risk factors for preterm birth in Japan: A multicenter, prospective study. 2009.

24.L. Guedes-Martins, E. Valente, L. Lima, J. Saraiva, A. Cunha. Second trimester down syndrome . Maternal serum screening in twin pregnancies: a retrospective study of 157 cases. 